\title{
Sensitive and Accurate Determination of Copper in Blood and Urine Samples by Vortex-assisted Dispersive Liquid-liquid Microextraction-slotted Quartz Tube - Flame Atomic Absorption Spectrometry After Complexation with a Novel Schiff-base Ligand
}

\author{
Emine Gülhan Bakirdere ${ }^{a, *}$ and Esra Maltepe ${ }^{b}$ \\ a Yildiz Technical University, Faculty of Education, Department of Science Education, \\ 34210 Istanbul, Turkey \\ b Yildiz Technical University, Faculty of Art and Science, Chemistry Department, \\ 34210 Istanbul, Turkey
}

\section{INTRODUCTION}

Copper plays an important role in regulating the DNA/RNA transcription processes, the metabolism of nitrogen-containing compounds and plant photosynthesis, thus, it is referred to as a biogenic element which is essential for both plants and animals (1-3). Copper is widely used in products such as fertilizers, wood preservatives, pesticides and water distribution systems (pipes) (4). Activities such as mining and industrial waste disposal, combustion of unrefined or contaminated gasoline could lead to the release of this element into the environment $(5,6)$. It was reported that copper enters and accumulates in aquatic plants, fish and other environmental organisms and since it moves up the food chain, it becomes biomagnified, and the organisms at the higher trophic levels become exposed to higher concentrations (7). The common routes for $\mathrm{Cu}$ exposure are through drinking water, eating food, inhaling air, and having direct skin contact with the metal (8). At high exposure levels, copper causes health disorders such as diarrhea, nausea, kidney damage, liver damage, even death under extreme conditions (8). Lethal doses of copper in adults range from 4.0 - $400 \mathrm{mg}$ per kilogram of body weight as

\footnotetext{
* Corresponding author.

E-mail: gulhan063@gmail.com

Tel: +902123834896
}

\section{ABSTRACT}

Due to the low sensitivity of flame atomic absorption spectrometry for trace copper determination, dispersive liquid-liquid microextraction and slotted quartz tube were used to increase the instrument's detection power by about 102 -fold. This high detection improvement was achieved by performing a comprehensive optimization study on the microextraction method and slotted quartz tube parameters.

A novel ligand was used to form the copper complex prior to the extraction process. The limits of detection and quantification obtained under optimum experimental conditions were 0.52 and $1.7 \mathrm{ng} / \mathrm{mL}$, respectively. The calibration plot was linear over a wide concentration range, and the percent relative standard deviation of the lowest concentration in the linear calibration plot was less than $5.0 \%$, indicating high precision for the extraction procedure and instrumental measurements.

In order to determine the validity and applicability of the developed method, recovery studies were carried out on blood and urine samples at different spiked concentrations. The recovery results recorded for both samples ranged between $85 \%$ and $113 \%$ which confirmed that the method can be applied to these matrices with high accuracy and precision. stated by the World Health Organization for suicidal and accidental ingestion (9). Due to the low levels of copper in the environment, sensitive analytical techniques are required for its accurate and precise determination.

The complex nature of environmental and biological sample matrices makes direct instrumental analysis difficult as the matrix components could affect the results or cause harm to the instrument. Sample preparation is, therefore, used to obtain the analyte in a clean and compatible form for instrumental reading, with extraction being a commonly used method. Recently developed microextraction methods are simple, fast, require low amounts of toxic solvent, result in low analyte loss, high enrichment factors, and high analytical recovery (10). Methods such as singledrop microextraction (SDME), solidified floating organic microextraction (SFODME), solid phase microextraction (SPME), and hollow fiber liquid phase microextraction (HF-LPME) have been used to preconcentrate copper and other metals for trace determination (1114). Dispersive liquid-liquid microextraction (DLLME) is an important method that was introduced by Rezaee et al. in 2006 (15), and it has since become a widely used method for both organic and inorganic analyses. DLLME employs dispersive solvents that are miscible with both an aqueous sample and 
an extractant, and this miscibility ensures a fine distribution of extraction solvent and increases extraction surface area. Extraction is completed in a few seconds, and the high surface area results in high extraction output (16). The extract after DLLME is compatible with several instrumental techniques used for qualitative and quantitative determination of the analytes.

Modern analytical instruments have been conveniently used over the classical gravimetric and titrimetric methods due to a rapid, accurate, and precise analysis. Inductively coupled plasma (ICP) techniques, equipped with mass spectrometry (ICP-MS), and optical emission spectrometry (ICP-OES) detection systems offer multi-elemental analysis and very low detection limits for several metals ( 17 , 18). However, these plasma techniques tend to be relatively expensive in terms of instrument and operational cost. Atomic absorption spectrometry (AAS) techniques, such as graphite furnace (GFAAS) and hydride generation (HG-AAS), have good sensitivities and are comparable to the plasma techniques, but they can only be used to determine one analyte at a time, and HG-AAS is only applicable to hydride-forming elements such as $\mathrm{Te}, \mathrm{Pb}, \mathrm{Sb}, \mathrm{Se}$, and As (19). Flame atomic absorption spectrometry (FAAS) is a very common metal detetection technique due to its robustness, ease of operation, and relatively low instrument cost (10). The nebulizer unit of the FAAS is considered its weakest point because only a fraction $(<10 \%)$ of aspirated sample reaches the flame for atomization, and this results in low sensitivity for most metals (16). A basic external component attached to the flame unit to improve absorbance measurements of the FAAS is a slotted quartz tube (SQT). The SQT works on two principles: increasing residence time of the atoms in the flame, leading to a 2-5-fold enhancement in absorbance (Figure 1), and trapping atoms within the inner walls of the SQT for the subsequent sudden release to obtain a sharp analytical signal (20).

The main purpose of this study was to develop an analytical method for the determination of copper at trace levels in blood and urine samples by slotted quartz tube-flame atomic absorption spectrometry after dispersed liquidliquid microextraction. The complexation of copper for extraction was achieved using a ligand synthesized in our laboratory (21) and all variables were systematically optimized to improve the analytical signal of copper.

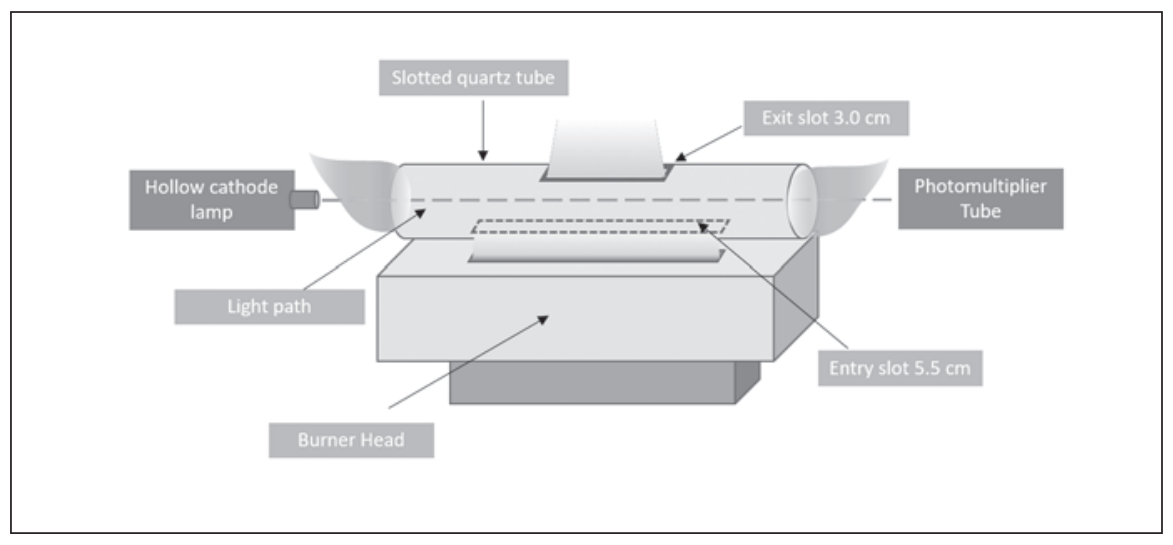

Fig. 1. Schematic diagram of FAAS burner bead with an attached SQT (16).

\section{EXPERIMENTAL}

\section{Instrumentation}

All absorbance measurements were performed with a NovAA (Analytik Jena AG, Germany) FAAS system. The flame was generated with a mixture of air and acetylene, and the flow rate of the acetylene was optimized to enhance the atomization efficiency. The $324.8 \mathrm{~nm}$ analytical line of $\mathrm{Cu}$ was selected from a multi-element hollow cathode lamp (Fe, Co, Ni, Mn, Cu, Cr) operating at $6.0 \mathrm{~mA}$ current and $0.50 \mathrm{~nm}$ spectral bandpass. A deuterium lamp $\left(\mathrm{D}_{2}\right)$ was used as background correction for all measurements. A $140 \mathrm{~mm}$ long quartz tube was used in this study which was cut at two different sides (entry and exit slots) at an angle of $180^{\circ}$. The lengths of the entry and exit slots were $55 \mathrm{~mm}$ and $30 \mathrm{~mm}$, respectively. The SQT-FAAS system is represented in Figure 1 (16). Other instruments used in the study were EBA20 Andreas Hettich centrifuge, M-100 model HAPA ultrasonicator, Kermanlar mechanical shaker, and a HI2020 Hanna Instruments pH meter.

\section{Reagents and Standard Solutions}

Appropriate aliquots from a $1000 \mathrm{mg} / \mathrm{L}$ copper stock standard solution (purchased from High Purity Standards) were diluted to prepare working standards and calibration standard solutions. Ultrapure deionized water (resistivity 18.2 $\mathrm{M} \Omega \cdot \mathrm{cm}$ ) was used for dilution of the standards and for general cleaning purposes. All chemicals and reagents used in this study were of analytical grade. Optimization of the extraction method was carried out with methanol, ethanol, chloroform, 2-propanol, carbon tetrachloride, dichloromethane, 1,2-dichloroethane, sodium chloride, potassium chloride, sodium sulfate and potassium nitrate, all purchased from Merck (Darmstadt, 
Germany). Also used were ligand chemicals.

\section{Ligand Synthesis}

In the synthesis of the ligand, the procedure listed in the literature was slightly modified (21). 3-methoxy-5-bromosalicylaldehyde (10 mmol $2.31 \mathrm{~g}$ ) and $p$-toluenesulfonic acid (0.01 g) were dissolved in $25 \mathrm{~mL}$ of ethyl alcohol. 1,4phenylenediamine $(20 \mathrm{mmol}$, $2.16 \mathrm{~g}$ ) dissolved in $25 \mathrm{~mL}$ of ethyl alcohol was slowly added dropwise under reflux for 1.0 hour at $60{ }^{\circ} \mathrm{C}$. The aldehyde carbonyl group was followed by IR. The dark orange product obtained as a result of the reaction was allowed to stand overnight and then filtered off from the solvent. The product was washed several times with hot distilled water, ethyl alcohol, and diethylether. The resulting product was then dried under vacuum and stored in a desiccator. Characterization of the synthesized ligand $\left(\mathrm{C}_{14} \mathrm{H}_{13} \mathrm{O}_{2} \mathrm{~N}_{2} \mathrm{Br}\right)$ was performed.

\section{Procedure of DLLME}

$1.0 \mathrm{~mL}$ of $\mathrm{pH} 8$ buffer solution and $0.50 \mathrm{~mL}$ of ligand solution $(0.02 \% \mathrm{w} / \mathrm{v})$ were consecutively added to $8.0 \mathrm{~mL}$ of standard/sample solution. Mixing by hand-shaking for 15 seconds was done to ensure uniform distribution of the ligand throughout the aqueous solution. A mixture of 2-propanol $(3.0 \mathrm{~mL})$ and chloroform $(300 \mu \mathrm{L})$ was then injected into the complex solution, resulting in a cloudy solution. After 15 seconds of vortexing, the solution was centrifuged at $6000 \mathrm{rpm}$ for 2.0 minutes. Finally, the extracted phase was taken into a separate tube for complete evaporation of the chloroform. The residue was dissolved with $200 \mu \mathrm{L}$ concentrated $\mathrm{HNO}_{3}$ and sent to the FAAS for measurements. An amount of 0.2 $\mathrm{mL}$ is enough to get a sharp analytical signal in FAAS.

\section{RESULTS AND DISCUSSIONS}

Step-by-step optimizations were carried out for the complex formation and DLLME to enhance the sensitivity for the Cu determination. In the optimization of one parameter, the other parameters were held at constant values, and the highest mean absorbance of triplicate measurements was used to determine the optimum values. Optimization studies were performed with appropriate aqueous standards with absorbance values within the linear calibration range.

\section{Complex Formation Optimization}

Parameters varying in this optimization step were $\mathrm{pH}$ and amount of buffer solution, ligand concentration and ligand amount. Buffer solutions are added to sample solutions in order to provide a favorable medium for complexation and maintain the stability of the complex. For this reason, buffer solutions with $\mathrm{pH}$ values ranging from 5 to 11 were tested for their effects on the Cu complex formation. The absorbance values recorded increased linearly from $\mathrm{pH} 5$ up to 8 , after which it decreased marginally. A pH 8 buffer solution was favorable for the complex formation because an acidic medium pre-

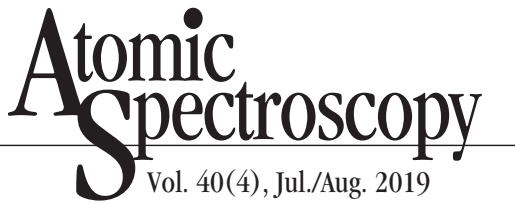

sents free hydroxonium ions which compete with complexation, and a highly basic medium could lead to deprotonation of the ligand's hydroxyl groups (22). The volume of buffer solution used is also important because an excess amount could result in analyte dilution. The optimum amount of $\mathrm{pH} 8$ buffer solution was determined by testing $0.50,1.0,1.5$, and $2.0 \mathrm{~mL}$ volumes. The absorbance values recorded were not different from each other, thus, $0.50 \mathrm{~mL}$ was selected in order to lower the use of chemicals.

The concentration of ligand solution is another important factor for complexation, and this was optimized by testing $0.02,0.065$, and $0.10 \%(\mathrm{w} / \mathrm{v})$ ligand concentrations. The absorbance signals recorded were not significantly different from each other, but $0.065 \%$ was selected for being slightly higher than the other two concentrations. The optimum amount of the ligand required for efficient complexation of $8.0 \mathrm{~mL}$ sample/standard solution was determined by testing $0.25,0.50,1.0$, 1.5 , and $2.0 \mathrm{~mL}$ volumes. The absorbance values increased steadily from $0.25 \mathrm{~mL}$ up to $1.5 \mathrm{~mL}$, after which it plateaued as is shown in Figure 2. For an even distribution

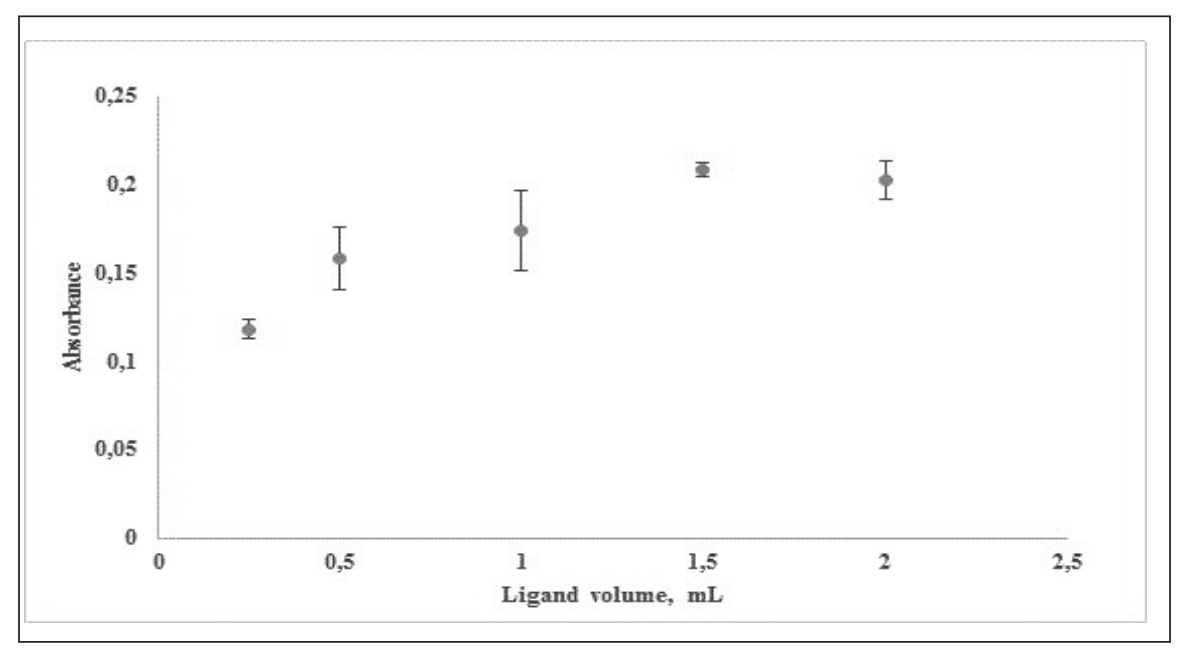

Fig. 2. The graphic of ligand volume optimization. 
of ligand throughout the aqueous solution, 15 seconds of vortexing was employed in this study. Some studies in the literature reported that excess mixing periods lower the absorbance signals $(10,22)$ and for this reason, mixing during complexation was not optimized.

\section{Optimization of Extraction Solvent Type and Volume}

An ideal extraction solvent should be immiscible with water and should extract an appreciable amount of analyte(s). The centrifuge tubes $(15 \mathrm{~mL})$ used in the study had a tapered bottom which was convenient for collecting the microliter volume of extraction solvents used. For this purpose, only high density solvents ( $p>1.0$ ) including carbon tetrachloride, 1,2-dichloroethane, chloroform, and dichloromethane were tested for their extraction efficiencies on the copper complex. $500 \mu \mathrm{L}$ of each solvent was mixed with 3.0 $\mathrm{mL}$ of 2-propanol and injected in separate extractions. Chloroform recorded the highest average absorbance as well as the least relative standard deviation value. A very low amount of extraction solvent might not be sufficient to extract appreciable amounts of analyte and an excess amount could make processing of the extract for instrumental read-out difficult. The optimum amount of chloroform was determined by testing 200 , $250,300,400$, and $500 \mu \mathrm{L}$ volumes of chloroform under similar conditions. Absorbance signals increased marginally from 200 to $300 \mu \mathrm{L}$, and then decreased marginally again at the higher volumes. The optimum chloroform volume for further optimizations was therefore set at $300 \mu \mathrm{L}$

\section{Optimization of Dispersive Solvent Type and Volume}

Low carbon chain alcohols have mid polarity index values which makes them miscible with most polar and apolar organic solvents. This miscibility makes them ideal for the role of dispersive solvents in DLLME $(23,24)$. Ethanol, methanol, and 2-propanol are all miscible with chloroform, and $3.0 \mathrm{~mL}$ of each was mixed with $300 \mu \mathrm{L}$ of chloroform, then tested for their dispersive efficiencies. 2-propanol recorded the highest average absorbance for triplicate measurements and was approximately 2-3 times higher than methanol and ethanol, respectively (Figure 3). The degree of extractant dispersion depends on the volume of the dispersive solvent used, and the optimum volume of 2-propanol was determined by testing $1.5,2.0,2.5$, and $3.0 \mathrm{~mL}$. The absorbance value increased gradually from $1.5 \mathrm{~mL}$ up to $3.0 \mathrm{~mL}$ as shown in Figure 4 . The 2-pro-panol volumes higher than $3.0 \mathrm{~mL}$ were not tested because their sum total with the volumes of sample, ligand, and buffer solution would have exceeded the volume of the tube used. A significant increase would not be expected for higher volumes as the results can be seen to have plateaued. $3.0 \mathrm{~mL}$ was therefore selected as optimum volume of 2-propanol.

\section{Salt Effect}

In liquid-liquid extraction methods, salting out of analyte(s) is achieved by adding highly soluble inorganic salts to aqueous solutions. The salts displace analytes in the aqueous medium, thereby enhancing their mass transfer into the extractant. However, an appropriate amount of the salt needs to be added in order to avoid saturation which could hinder the extraction of the analytes. For extraction solvents, which have

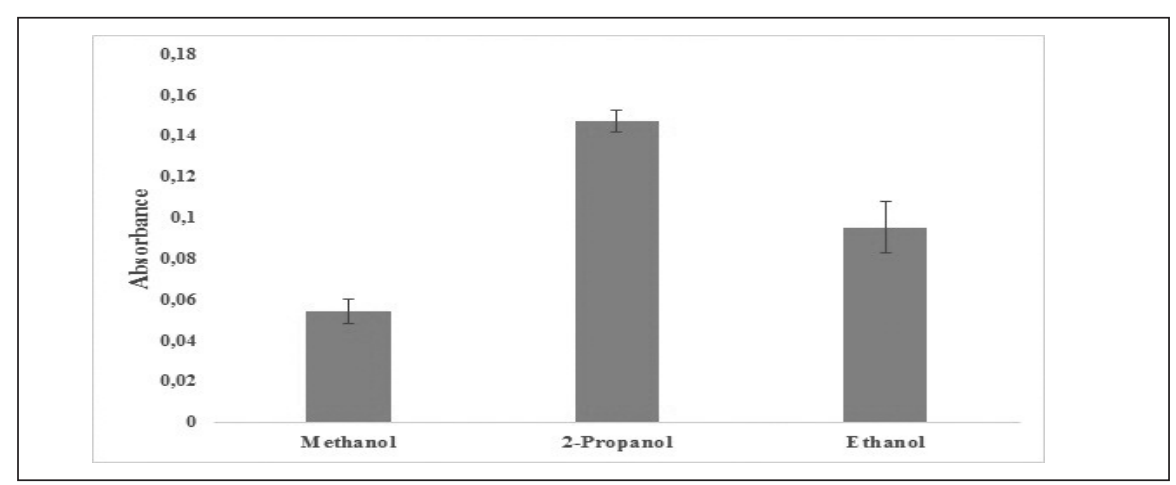

Fig. 3. A chart of dispersive solvents against average absorbance values ( $n=3$ error bars).

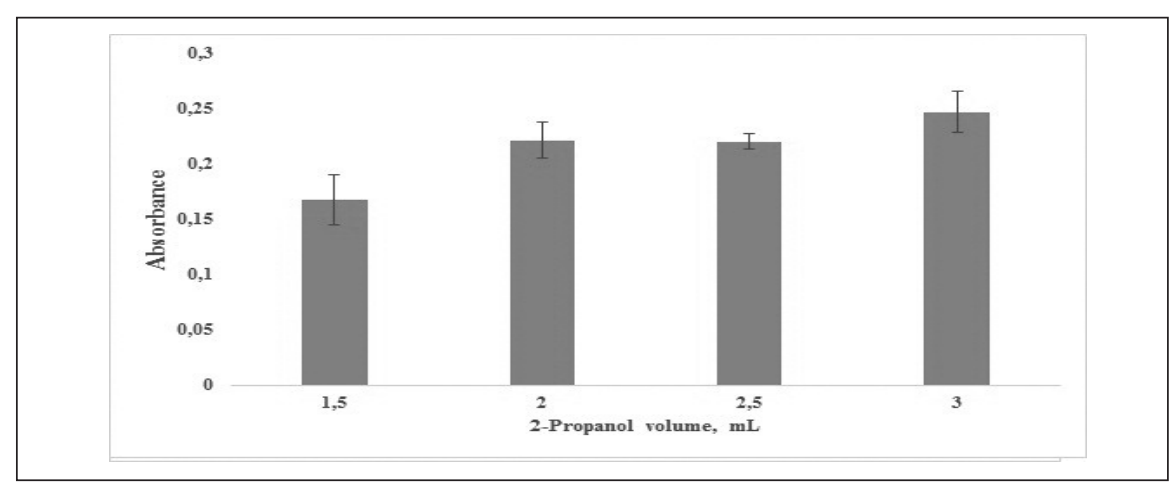

Fi. 4. A chart of 2-propanol volumes against average absorbance values ( $n=3$ error bars). 
densities very similar to the sample solution, addition of a salt prior to extraction could alter the density of the solution and result in a better phase separation (10). For these reasons, $1.0 \mathrm{~g}$ each of $\mathrm{NaCI}, \mathrm{KNO}_{3}$, $\mathrm{Na}_{2} \mathrm{SO}_{4}$, and $\mathrm{KCl}$ were totally dissolved in equivalent aqueous standards, and their extraction results were compared to salt-free extraction. Although the highest yields were obtained with $\mathrm{KCl}$, it was not significantly higher than the other salt-added extractions. The $\mathrm{KCl}-$ added extraction was about $\mathbf{1 . 1}$ times higher than the salt-free extraction, and this enhancement was not deemed significant to continue further extractions with $\mathrm{KCl}$.

\section{Analytical Performance}

Table I is a summary of the optimal experimental parameters, and using these parameters, the analytical performance of all systems were determined using calibration plots. Table II summarizes the figures of merit calculated for FAAS, SQTFAAS, DLLME-FAAS, and DLLMESQT-FAAS. A comparison of these systems to other literature studies is also presented in Table II. The calibration plots were linear over wide concentration ranges, and the regression coefficients $\left(R^{2}\right)$ were greater than 0.9993. The limits of detection (LOD) and quantification (LOQ) were determined using the following equations:

$$
\begin{aligned}
& \mathrm{LOD}=3 \text { Stdev } / \mathrm{m} \\
& \mathrm{LOQ}=10 \text { Stdev } / \mathrm{m} \quad \text { (Eq. } 1)
\end{aligned}
$$

The standard deviation (Stdev) was calculated from six replicate absorbances of the lowest concentrations of each calibration plot, and the slope (m) was taken from the linear regression equation.

TABLE I

Optimized Parameters of DLLME-SQT-FAAS

\begin{tabular}{ll}
\hline Parameters & Value \\
\hline pH of buffer solution (amount) & $\mathrm{pH} 8.0(1.0 \mathrm{~mL})$ \\
Ligand concentration (amount) & $0.02 \%, \mathrm{w} / \mathrm{v}(0.50 \mathrm{~mL})$ \\
Complexing period & $15 \mathrm{~s}$ (Vortex) \\
Extraction solvent (amount) & Chloroform $(300 \mu \mathrm{L})$ \\
Dispersive solvent (amount) & 2 -Propanol $(3.0 \mathrm{~mL})$ \\
Sample flow rate* & $6.44 \mathrm{~mL} / \mathrm{min}$ \\
Acetylene flow rate* $^{*}$ & $40 \mathrm{~L} / \mathrm{hr}$ \\
SQT height* & $0.0 \mathrm{~mm}$ \\
\hline
\end{tabular}

* Parameters taken from Özzeybek et al. (10).

TABLE II

Analytical Figures of Merit for Different FAAS Systems Under Study

\begin{tabular}{lccccc}
\hline Analytical Method & $\begin{array}{c}\text { LOD } \\
(\mathrm{ng} / \mathrm{m}\end{array}$ & $\begin{array}{c}\text { LOQ }, \\
(\mathrm{ng} / \mathrm{mL})\end{array}$ & $\begin{array}{c}\text { Range } \\
(\mathrm{ng} / \mathrm{mL})\end{array}$ & \multicolumn{2}{c}{ RSD } \\
$(\%)^{\mathrm{a}}$ & & \\
\hline FAAS & 53 & 177 & $200-10,000$ & 5.5 & \\
SQT-FAAS & 23 & 75 & $50-1000$ & 8.4 & \\
DLLME-FAAS & 1.4 & 4.6 & $5.0-500$ & 8.9 & \\
DLLME-SQT-FAAS & 0.52 & 1.7 & $2.0-250$ & 3.6 & \\
DLLME-SQT-FAAS & 0.7 & 2.2 & $2.0-100$ & 4.7 & $(10)$ \\
DLLME-FAAS & 1.9 & - & $8.0-400$ & 3.8 & $(25)$ \\
\hline
\end{tabular}

*For FAAS and SQT-FAAS systems, the values of LOD and LOQ taken from Özzeybek et al. (10).

\section{Atomic Apectroscopy 1 Vol. 40(4), Jul./Aug. 2019}

According to the LOD values, increases in detection power with respect to FAAS were 2.3-, 38-, and 102-fold for SQT-FAAS, DLLMEFAAS, and DLLME-SQT-FAAS, respectively.

The LOD and LOQ values for the optimum system (DLLME-SQTFAAS) were found to be 0.52 and $1.7 \mathrm{ng} / \mathrm{mL}$, respectively. The precision of the extraction procedure and the instrumental measurement was appreciably high as all measurements recorded percent relative standard deviations lower than $10 \%$.

\section{Recovery}

A recovery study was conducted to test the accuracy and applicability of this method to biological samples. It is a fact that heavy metals are not easily removed from the human body. For this reason, it is very important to be able to perform heavy metal determination at trace levels in biological samples such as urine and blood. These biological samples have very complex matrices which affects the accurate quantification of the analytes. The acid digestion procedure reported by Erarpat et al. (16) was therefore used to decompose the organic matrix, leaving the metal (inorganic) component for analysis. The solution after digestion was diluted with deionized water after evaporating to near dryness which was repeated several times. Then, 1.0 $\mathrm{mL}$ of $\mathrm{pH} 8$ buffer solution was added to the solution to adjust the $\mathrm{pH}$ of the solution to 8.0. The sample analysis was then performed, and the absence of copper signals suggested that the analyte was not present in the samples according to the limit of detection. The digested blood and urine samples were selectively spiked to final concentrations of 25 and $50 \mathrm{ng} / \mathrm{mL}$ and analyzed under optimum experimental conditions. The reason for the selection of the stated concentrations was that they were within 
the linear calibration range, and gave distinct signals. The recovery results obtained using aqueous calibration standards ranged between 85 and $113 \%$ for both samples as given in Table III. The precision of the method for the biological matrices was also confirmed by low $\%$ RSD values $(<5.0 \%)$. These results were satisfactory and established that the method can be used for an accurate and precise quantification of copper in blood and urine samples.

TABLE III

Percent Recovery Results for Blood and Urine

\begin{tabular}{lrr}
\hline Sample & $25 \mathrm{ng} / \mathrm{mL}$ & $50 \mathrm{ng} / \mathrm{mL}$ \\
\hline Blood & $85.1 \pm 3.4$ & $112.9 \pm 4.2$ \\
Urine & $95.4 \pm 3.3$ & $110.6 \pm 3.1$ \\
\hline
\end{tabular}

\section{CONCLUSION}

In this study, a simple, accurate, and precise analytical method was developed for the determination of copper at trace level by FAAS. Dispersive liquid-liquid microextraction was comprehensively optimized to obtain a high preconcentration factor and slotted quartz tube was used to further enhance the detection power of the FAAS instrument. Under the optimum extraction and system conditions, the method was found to have an approximately 102-fold enhancement in detection power with a detection limit of $0.52 \mathrm{ng} / \mathrm{mL}$. Other figures of merit were very satisfactory, and applicability of the method was then tested for the urine and blood samples. Recovery results from the spiked tests were obtained in the range of $85-113 \%$ and confirmed that the method is appropriate for testing biological matrices. The developed method is economical, easy, rapid, and agrees with green chemistry which is aimed at reducing toxic chemical usage.

Declaration of interest: None.

Received February 5, 2019.

\section{REFERENCES}

1. I. Yruela, Metallomics 5(9), 1090 (2013).

2. J. Lakshmipraba et al., Spectrochim. Acta Part A: Molecular and Biomol. Spectrosc. 109, 23 (2013).

3. G. Güleryüz, Chemistry and Ecology 31(6), 494 (2015).

4. C. Mauceri and S. Mishra, Copper (Cu), in Health Effects of Metals and Related Substances in Drinking Water, M. Ferrante, O.C. Gea, and Z. Rasic-Milutinovic, Editors, IWA Publishing: London, UK (2013).

5. A. Tobiasz, Talanta 96, 82 (2012).

6. S.N. Do Carmo et al., Microchem. Journal 110, 320 (2013).

7. G.S. Rocha et al., Ecotoxicol. and Environmental Safety 133, 235 (2016).

8. V.A. Lemos et al., Environmental Monitoring and Assessment 148(1), 245 (2009).

9. WHO, Copper in drinking-water: Background document for development of WHO Guidelines for Drinking-Water Quality. World Health Organization, 2004 (WHO/SDE/WSH/03.04/88).

10. G. Özzeybek et al., Microchem. Journal 132, 406 (2017).

11. X. Wen, Q. Deng, and J. Guo, Spectrochim. Acta Part A: Molecular and Biomolecular Spectrosc. 79(5), 1941 (2011).

12. Ç.A. Sahin and İ. Tokgöz, Anal. Chim. Acta 667(1), 83 (2010).

13. S.A. Arain et al., J. of Anal. At. Spectrom. 29(12), 2362 (2014).

14. Z. Es'haghi and R. Azmoodeh, Arabian Journal of Chem. 3(1), 21 (2016).

15. M. Rezaee et al., J. of Chromatography A 1116(1), 1 (2006).

16. S. Erarpat et al., Chemosphere 189, 180 (2017).

17. M.C. Bruzzoniti et al., Talanta 99, 703 (2012).

18. J. Moreda-Piñeiro et al., Microchem. Journal 137, 402 (2018).

19. N.A. Kasa,et al., Microchem. J. 133, 144 (2017).
20. I. Demirtas, S. Bakirdere, and O.Y. Ataman, Talanta 138, 218 (2015).

21. E.G. Bakirdere, Synthesis and characterization of complexes produced from $\mathrm{N}_{2} \mathrm{O}_{2}$ type Schiff bases with $\mathrm{Co}(\mathrm{II}), \mathrm{Ni}(\mathrm{II}), \mathrm{Cu}$ (II) and $\mathrm{Zn}$ (II) metals in chemistry. Firat University, Turkey (2008).

22. M.S. Findikoglu et al., Water, Air, \& Soil Pollution 229(2), 37 (2018).

23. M. Firat et al., Spectrochim. Acta Part B: At. Spectrosc. 129, 37 (2017).

24. E. Öztürk Er, et al., J. of Trace Elements in Medicine and Biology 49, 13 (2018).

25. M. Rajabi et al., J. of Molecular Liquids 194, 166 (2014). 